\title{
Near-field diffraction of chirped gratings
}

\author{
Luis Miguel Sanchez-Brea, ${ }^{1, *}$ Francisco Jose Torcal-Milla, ${ }^{1}$ and Tomas Morlanes ${ }^{2}$ \\ 'Optics Department, Applied Optics Complutense Group, Universidad Complutense de Madrid, Facultad de Ciencias Físicas, \\ Plaza de las Ciencias 1, 28040 Madrid, Spain \\ ${ }^{2}$ Fagor Aotek S. Coop., B San Andrés 19, 20500 Mondragón, Guipuzcoa, Spain \\ *Corresponding author: sanchezbrea@fis.ucm.es
}

Received 7 July 2016; accepted 5 August 2016; posted 12 August 2016 (Doc. ID 269534); published 0 MONTH 0000

In this Letter, we analyze the near-field diffraction pattern produced by chirped gratings. An intuitive analytical interpretation of the generated diffraction orders is proposed. Several interesting properties of the near-field diffraction pattern can be determined, such as the period of the fringes and its visibility. Diffraction orders present different widths and also, some of them present focusing properties. The width, location, and depth of focus of the converging diffraction orders are also determined. The analytical expressions are compared to numerical simulation and experimental results, showing a high agreement. (๐) 2016 Optical Society of America

OCIS codes: (050.1940) Diffraction; (050.1950) Diffraction gratings; (070.6760) Talbot and self-imaging effects; (350.2770) Gratings; (050.1590) Chirping.

http://dx.doi.org/10.1364/OL.99.099999

When a diffraction grating is illuminated with a monochromatic plane wave, self-images are produced at multiples of the so-called Talbot distance, $z_{T}=2 p^{2} / \lambda$, with $p$ being the period of the grating and $\lambda$ the wavelength $[1,2]$. The Talbot effect has increased interest in many diverse fields [3]. It has been analyzed for several kinds of illumination, such as Gaussian beams [4], and for several kinds of diffraction gratings, such as metallic gratings [5], or imperfect diffraction gratings [6-9]. Self-images do not only appear for periodic objects (Talbot self-images) but also for quasi-periodic objects (Montgomery self-images) [10]. In the temporal range, chirped fiber gratings are used as a solution for dispersion compensation [11]. The focusing properties of these nonperiodic gratings have also been applied in third-generation synchrotron radiation and high-resolution $\mathrm{x}$ ray spectroscopy $[12,13]$. In the spatial range, chirped gratings have also been applied to produce curved diffraction orders [14]. Curved lobes are created by the caustic interference of the originally straight diffraction orders and manifest themselves as accelerating beams.

Since chirped gratings do not present a periodic structure, an analysis based on Fourier series and diffraction orders cannot be performed in a simple way. In this Letter, to determine the near-field intensity distribution produced by chirped gratings, we decompose the incident beam as a sum of narrow Gaussian beams so that the grating can be considered locally periodic for these narrow beams. Analytical expressions to explain amplitude and period of the fringes produced by this kind of nonperiodic grating are obtained, which are compared to numerical simulations and experimental results.

First, let us consider a monochromatic Gaussian light beam, with amplitude $u\left(x^{\prime}\right)=\exp \left(-x^{\prime 2} / \omega_{0}^{2}\right)$, which illuminates a chirped grating defined by its spatial frequency $q\left(x^{\prime}\right)$. The beam waist of the Gaussian beam is placed at the plane of the chirped grating, $z=0$. For simplicity, let us consider that the spatial frequencies of the grating present a linear dependency $q\left(x^{\prime}\right)=q_{0}+q_{a} x^{\prime}$. For the case of an amplitude binary grating, an example is shown in Fig. 1.

Now, let us divide the incident beam into a sum of narrow Gaussian beams, whose width is $\omega_{s}$ and placed at different

(a)

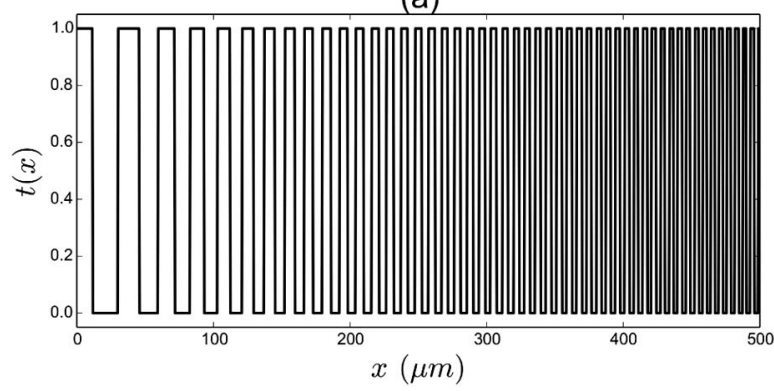

(b)

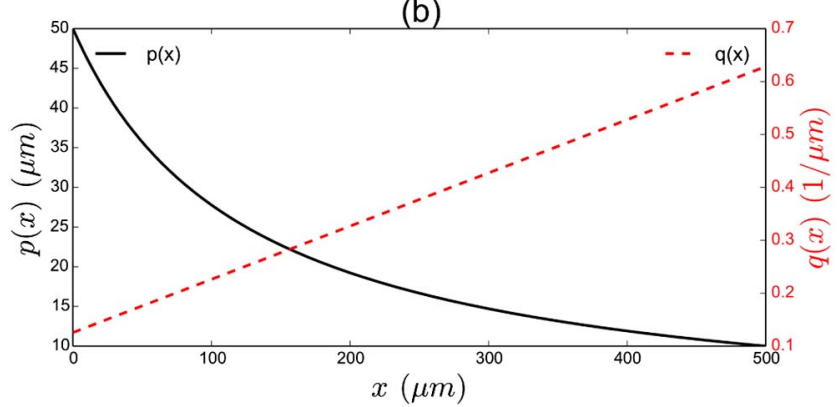

Fig. 1. (a) Chirped binary diffraction grating with starting period $p_{0}=50 \mu \mathrm{m}$, final period $p_{1}=10 \mu \mathrm{m}$, and length $l=500 \mu \mathrm{m}$. For this case, the spatial frequency dependency is linear: $q\left(x^{\prime}\right)=0.125+0.001 x^{\prime} \mu \mathrm{m}^{-1}$. (b) Period (black solid line) and spatial frequency (dashed red line) of the grating in terms of position. 
locations $x_{s}$. Considering that a plane wave can be described as a sum of narrow Gaussian beams, we can write $1=\int_{-\infty}^{+\infty} \exp \left[-\left(x^{\prime}-x_{s}\right)^{2} / \omega_{s}^{2}\right] \mathrm{d} x_{s} /\left(\sqrt{2 \pi} \omega_{s}\right)$. Besides, we can assume that for each beam placed at $x_{s}$, only a very narrow area of the grating is illuminated. Then, this narrow beam sees a periodic grating with constant frequency $q\left(x_{s}\right)=q_{0}+q_{a} x_{s}$. When the illumination is a Gaussian beam with amplitude $\exp \left(-x^{\prime 2} / \omega_{0}^{2}\right)$, the amplitude just after the grating results in

$$
\begin{aligned}
U\left(x^{\prime}\right) \propto & \exp \left(-\frac{x^{\prime 2}}{\omega_{0}^{2}}\right) \exp \left[-\frac{\left(x^{\prime}-x_{s}\right)^{2}}{\omega_{s}^{2}}\right] \\
& \times \sum_{n} a_{n} \exp \left[\operatorname{in}\left(q_{0}+q_{a} x_{s}\right) x_{s}\right],
\end{aligned}
$$

where $\sum_{n} a_{n} \exp \left[\operatorname{in}\left(q_{0}+q_{a} x_{s}\right) x_{s}\right]$ is the Fourier description of the local grating, $a_{n}$ are the Fourier coefficients, and $n$ are integers.

Then, considering the Fresnel approach for the near field, the amplitude at a distance $z$ produced by this narrow Gaussian beam is $U^{s}(x, z) \propto \int_{-\infty}^{+\infty} U\left(x^{\prime}\right) \exp \left[i k\left(x-x^{\prime}\right)^{2} / 2 z\right] \mathrm{d} x^{\prime}$, where $k=2 \pi / \lambda$ is the wavenumber. The amplitude produced by the whole beam, whose width is $\omega_{0}$, is obtained as the integral of all the narrow beams, considering that each beam has a negligible width, $\omega_{s} \rightarrow 0, U(x, z)=\operatorname{limit}_{\omega_{s} \rightarrow 0} \int_{-\infty}^{\infty} U^{s}(x, z) \mathrm{d} x_{s}$. This equation results in

$$
\begin{aligned}
U(x, z) \propto & \sum_{n} \frac{a_{n}}{\omega} \exp \left[-\frac{\left(x+n q_{0} z / k\right)^{2}}{\omega^{2}}\right] \exp \left(-\frac{i k x^{2}}{2 R}\right) \\
& \times \exp \left(\operatorname{in} \frac{k k_{p} \omega_{0}^{4}}{4 z R} q(x) x\right) \exp \left(\operatorname{in}^{2} \frac{k_{p} q_{0}^{2} \omega_{0}^{4}}{8 R}\right),
\end{aligned}
$$

where $k_{p}=k-2 n q_{a} z, \omega^{2}=\omega_{0}^{2}\left[\left(k_{p} / k\right)^{2}+\left(z / z_{R}\right)^{2}\right]$ is the beam width of each diffraction order, $R=z\left[\left(k_{p} / k\right)^{2}\left(z_{R} / z\right)^{2}+\right.$ $1]$ is the radius of curvature, and $z_{R}=k \omega_{0}^{2} / 2$ is the Rayleigh distance. Notice that both $R$ and $\omega$ parameters depend on the diffraction order $n$. The first exponential term of Eq. (2) represents the amplitude of the beam, which is Gaussian. Diffraction orders propagate with an angle $\theta_{p}=x / z=$ $n \lambda / p$ with respect to the axis, and the width $\omega$ of each diffraction order is different, since $k_{p}$ presents a dependence on $n$. The second term is the phase of the Gaussian beam. The third term is related to the period of the fringes, and the fourth term is related to the location of high contrast fringes.

In Fig. 2, we can see the intensity distribution in the near field along the $z$-axis, $I=U(x, z) U^{*}(x, z)$, for a chirped diffraction grating computed with Eq. (2). The fringes' period depends on the position $x$. To check the consistency of the results, we have also computed the near-field intensity by numerical simulations based on the Rayleigh-Sommerfeld method for diffraction [15], Fig. 3. There is a good agreement between the theoretical and the numerical approach. Also, the difference between the theoretical and numerical results, $\Delta I=I_{\text {teo }}-I_{\text {num }}$, has been evaluated. For this comparison, we have eliminated the edges of the diffraction pattern, since edges affect the numerical propagation of the grating. The difference between both approaches is less than $5 \%$ on average, which shows the validity of Eq. (2).

In a first stage, let us determine the amplitude of the fringes from Eq. (2) when the incident wave is plane. This can be obtained considering $\omega_{0} \rightarrow \infty$, which results in $U(x, z) \propto \sum_{n} a_{n} \exp \left[\operatorname{in}\left(k / k_{p}\right) q(x) x\right] \exp \left[\operatorname{in}^{2} k_{p} q_{0}^{2} z /\left(2 k^{2}\right)\right] . \quad W e$ can expand $k_{p}=k-2 n q_{a} z$ in both exponential terms. For

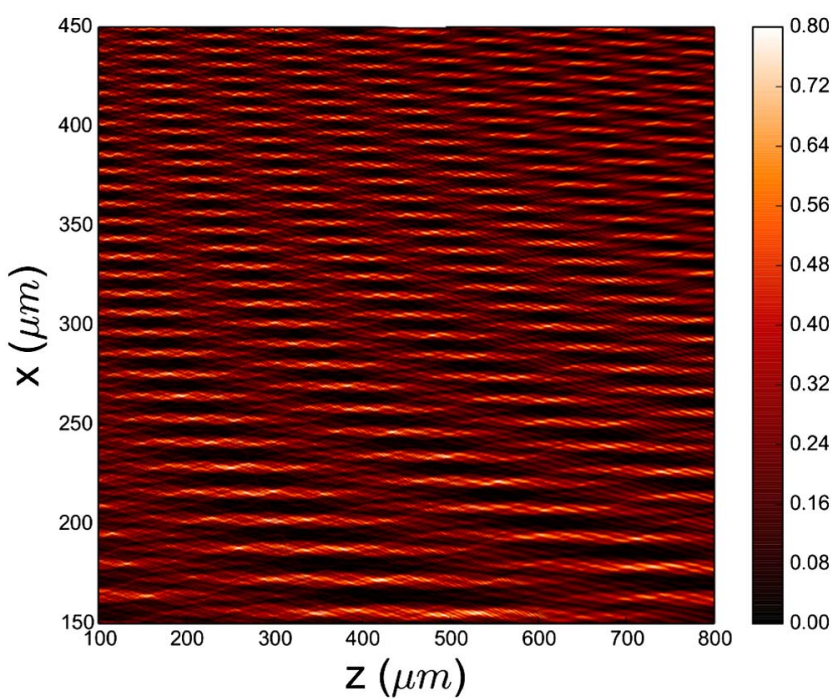

Fig. 2. Intensity distribution $I_{\text {teo }}$ obtained with Eq. (2) for a chirped grating with $p_{0}=80 \mu \mathrm{m}, p_{1}=10 \mu \mathrm{m}$, and length $l=600 \mu \mathrm{m}$. The grating is illuminated with a Gaussian beam whose wavelength is $\lambda=0.6328 \mu \mathrm{m}$; the beam width is $\omega_{0}=5000 \mu \mathrm{m}$, and $n=5$.

the first exponential, considering that $1 /\left(1-2 n q_{a} z / k\right) \approx$ $1+2 n q_{a} z / k$, we obtain

$$
U(x, z) \propto \sum_{n} a_{n} \exp [\operatorname{in} q(x) x] \exp \left[i \frac{n^{2}}{2 k}\left(q_{0}^{2}+4 q(x) q_{a} x\right) z\right] .
$$

Then, the period of the fringes results in

$$
p(x)=\frac{2 \pi}{q(x)}=\frac{2 \pi}{q_{0}+q_{a} x} .
$$

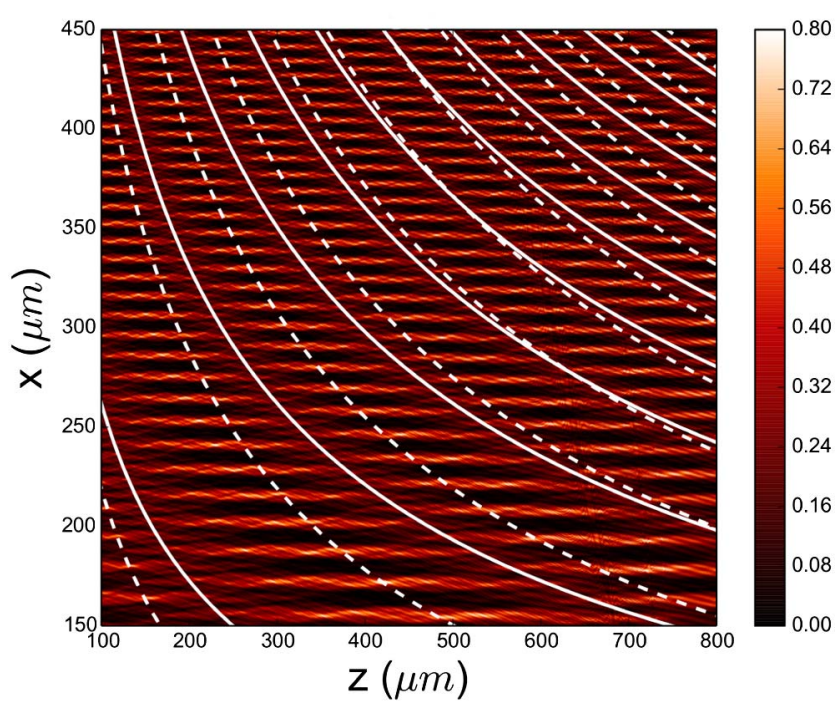

Fig. 3. Intensity distribution $I_{\text {num }}$ obtained by numerical Rayleigh-Sommerfeld approach [15] for the same parameters as Fig. 2. White continuous lines represent low-contrast positions obtained with Eq. (5) and white discontinuous lines represent lowcontrast positions according to $(l+1 / 2) p(x)^{2} / \lambda$, with $p(x)$ defined in Eq. (4), and $l$ an integer.
113

F2:1

$\mathrm{F} 2: 2$

F2:3

F2:4

114

115

F3:1

F3:2

F3:3

F3:4

F3:5

F3:6 
This means that the period of the fringes is, for the approximation considered, a local phenomenon, since it is equal to the period of the grating at the same position $x$. On the other hand, we compare the second exponential term to $\exp \left(2 \pi i z / z_{T}\right)$ for the Talbot distance, resulting in

$$
z_{T}=\frac{8 \pi^{2}}{\lambda\left(q_{0}^{2}+4 q_{a} q_{0} x+4 q_{a}^{2} x^{2}\right)},
$$

which turns to $z_{T}=2 p^{2} / \lambda$ when $q_{a}=0$. In Fig. 3 , the locations of null contrast, $(l+1 / 2) z_{T}$, according to Eq. (5) are shown as white solid lines. Also, for comparison, locations of the low contrast fringes computed using $(l+1 / 2) p(x)^{2} / \lambda$, considering Eq. (4), are included as white discontinuous lines. It is clear that this last simple equation is not valid for determining the location of low contrast fringes, as can be observed in Fig. 3.

From the definition of $k_{p}$, after Eq. (2), we can see that when the grating is not chirped, $q_{a}=0$; then $k_{p}=k$ and the amplitude after the diffraction grating results in

$$
\begin{aligned}
U(x, z) \propto & \sum_{n} \frac{a_{n}}{\omega^{\prime}} \exp \left[-\frac{\left(x+n q_{0} z / k\right)^{2}}{\omega^{\prime 2}}\right] \exp \left(-\frac{i k x^{2}}{2 R^{\prime}}\right) \\
& \times \exp \left(i \frac{n \omega_{0}^{4} k^{2} q_{0} x}{4 z R^{\prime}}\right) \exp \left(i \frac{n^{2} k q_{0}^{2} \omega_{0}^{4}}{8 R^{\prime}}\right),
\end{aligned}
$$

where, for this case, $\omega^{\prime 2}=\omega_{0}^{2}\left[1+\left(z / z_{R}\right)^{2}\right]$ and $R^{\prime}=z\left[\left(z_{R} / z\right)^{2}+1\right]$. That is, we recover the standard selfimaging process for Gaussian beams [5].

Let us analyze the properties of the fringes formed by chirped gratings when the incident beam is Gaussian. When comparing Eq. (2) with Eq. (6), we may think that the third exponential term is related to the period of the fringes and the fourth term indicates the location of high contrast fringes, since it presents a dependence with $n^{2}$. However, this is not exactly the case. In order to determine the location of high-contrast fringes, we need to expand all the phase terms, since $k_{p}$ presents a dependence with $n$. This expansion results in

$$
\begin{aligned}
U(x, z) \propto & \sum_{n} a_{n} \exp \left[-\frac{\left(x+n q_{0} z / k\right)^{2}}{\omega^{2}}\right] \exp \left(-\frac{i k x^{2}}{2 R}\right) \\
& \times \exp \left[\operatorname{in} \frac{k^{2} \omega_{0}^{4}}{4 z R} q(x) x\right] \exp \left[\operatorname{in}^{2} \frac{k \omega_{0}^{4}}{2 R}\left(\frac{q_{0}^{2}}{4}-q_{a} q(x) x\right)\right] \\
& \times \exp \left(-\operatorname{in}^{3} \frac{q_{a} q_{0}^{2} \omega_{0}^{4} z}{4 R}\right),
\end{aligned}
$$

where the radius of curvature is, expanding the definition in Eq. (2), $R=z_{R}^{2} / z-4 n q_{a} z_{R}^{2} / k+\left[\left(2 n q_{a} z_{R} / k\right)^{2}+1\right] z$. Then, when the grating is illuminated with a Gaussian beam, the period is obtained comparing the third exponential term to $\exp [2 \pi i x / p(x)]$, resulting in

$$
p(x)=\frac{8 \pi z R}{k^{2} \omega_{0}^{4} q(x)} .
$$

This equation is not easy to be solved, since the radius of curvature $R$ depends on $n$ and $z$. However, it simplifies for short distances to the grating. When the governing term in the radius of curvature is $R \approx z_{R}^{2} / z$, the period is again that obtained with Eq. (4). To determine the location of highcontrast fringes, we will consider the quadratic term with $n$, $\exp \left[\operatorname{in}^{2} \frac{k \omega_{0}^{4}}{2 R}\left(q_{0}^{2} / 4+q_{a} q(x) x\right)\right]$ in Eq. (7). Comparing this term with $\exp \left(2 \pi \operatorname{in}^{2} z / z_{T}\right)$, we obtain the Talbot distance from this equation:

$$
\left.\pi k R\right|_{z=z_{T}}=z_{R}^{2}\left[q_{0}^{2} / 4+q_{a} q(x) x\right],
$$

which is a nonlinear equation, since $R$ presents a dependence with $z$. Again, when we use the previous approximation for the near field, $R \approx z_{R}^{2} / z$, the results simplify to Eq. (5).

Now, let us determine the width of the diffraction orders. Since the illumination beam is Gaussian, there is a distance from which the different diffraction orders do not interfere. This distance corresponds to $z_{n}=p \omega_{0} / 2 \lambda$, which can be easily obtained from a geometrical analysis [5]. After this distance, we can clearly see the Gaussian shape of the diffraction orders. The width of diffraction orders is defined in Eq. (2). Expanding $\omega$ in terms of $n$ we obtain

$$
\omega^{2}=\omega_{0}^{2}\left\{\left[1+\left(\frac{2 z}{k \omega_{0}^{2}}\right)^{2}\right]-\frac{2 z q_{a}}{k} n+\left(\frac{2 z q_{a}}{k}\right)^{2} n^{2}\right\} .
$$

It is surprising that this dependence presents a linear term with $n$, which means that the width of positive and negative diffraction orders is different. The width of positive diffraction orders decreases with respect to order $n=0$ and, on the contrary, the width of negative diffraction orders increases. This can be clearly seen in the numerical approach given in Fig. 4, where a Gaussian beam with wavelength $\lambda=0.650 \mathrm{~nm}$ and beam width $\omega_{0}=250 \mu \mathrm{m}$ is used for illuminating the chirped grating. Diffraction orders $n=1,3,5$, and 7 produced by the chirped grating are convergent. We can determine the location of the beam waist, $z_{\min }$, considering $d \omega^{2} / \mathrm{d} z=0$,

$$
z_{\min }=\frac{k n q_{a} \omega_{0}^{4}}{2\left(1+n^{2} q_{a}^{2} \omega_{0}^{4}\right)} .
$$

As a consequence, the beam waist of the diffraction orders is placed at the diffraction grating, $z_{\min }=0$, only for the order $n=0$. Besides, the location $x_{\min }$ of the beam waist can be

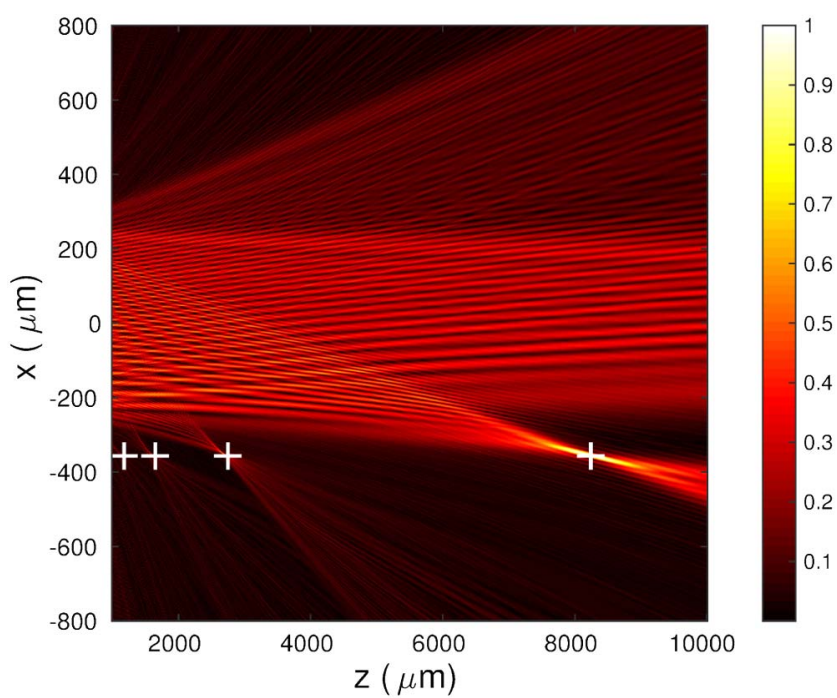

Fig. 4. Focusing properties of a chirped grating whose parameters are $p_{0}=50 \mu \mathrm{m}, p_{1}=15 \mu \mathrm{m}$, and length $l=500 \mu \mathrm{m}$. The grating is illuminated by a Gaussian beam with $\lambda=0.650 \mu \mathrm{m}$ and $\omega_{0}=250 \mu \mathrm{m}$. The white crosses represent the position of the beam waists $\left(x_{\min }, z_{\min }\right)$ computed using Eqs. (11) and (12). 182 


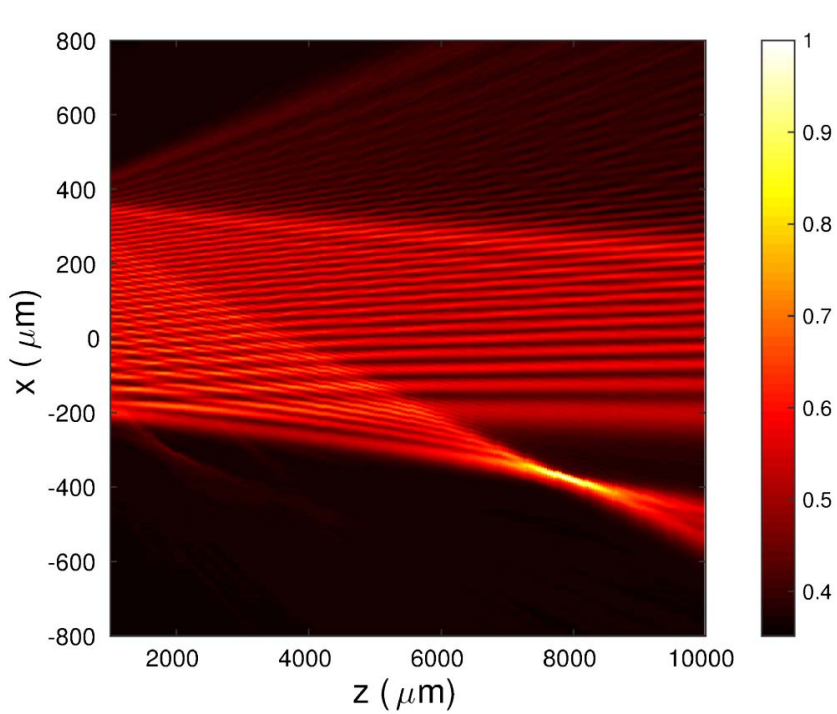

F5:1
Fig. 5. Experimental near-field intensity distribution for a chirped grating whose parameters are those of Fig. 4.

obtained from the first exponential term of Eq. (7), $\exp \left[-\left(x+n q_{0} z / k\right)^{2} / \omega^{2}\right]$, resulting in

$$
x_{\min }=\frac{n q_{0} z_{\min }}{k}=\frac{n^{2} q_{0} q_{a} \omega_{0}^{4}}{2\left(1+n^{2} q_{a}^{2} \omega_{0}^{4}\right)} \approx \frac{q_{0}}{2 q_{a}} .
$$

The white crosses in Fig. 4 represent the locations $\left(x_{\min }, z_{\min }\right)$ for these diffraction orders. Introducing Eq. (11) into Eq. (10), we obtain the beam waist of the diffraction orders:

$$
\omega_{\min }^{2}=\frac{\omega_{0}^{2}}{1+n^{2} q_{a}^{2} \omega_{0}^{4}} .
$$

This means that the beam width decreases with respect to the incident beam except for $n=0$. For example, we can see that the beam with $n=3$ is narrower than beam with $n=1$ (diffraction orders $n=5$ and 7 are very narrow and can hardly be observed due to their low power).

Another interesting parameter is the depth of focus of the beam waist. For Gaussian beams this parameter is measured by the Rayleigh distance $z_{R}=k \omega_{0}^{2} / 2$, which is the distance where the beam width is $\sqrt{2}$ times the width of the beam waist. Using this definition, we have found that the equivalent Rayleigh distance for the diffraction orders produced by the chirped grating is

$$
z_{R}^{\prime}=\frac{z_{R}}{1+n^{2} q_{a}^{2} \omega_{0}^{4}} .
$$

Diffraction orders present different depth of focus, and are shorter than for the case of the original beam width.

Finally, we have performed an experimental verification of the near-field intensity distribution after the chirped grating.
We have manufactured a chirped grating with the same parameters as in Fig. 4. The grating is illuminated by a monochromatic plane wave with wavelength $\lambda=0.650 \mu \mathrm{m}$. The experiment consists of acquiring the intensity distribution with a CMOS camera UI-3592LE by IDS Imaging Development Systems $\mathrm{GmbH}$, whose pixel size is $1.67 \mu \mathrm{m}$. The camera is placed on a motorized linear stage by PI, and it is moved along the $z$ axis, perpendicular to the grating. The experimental intensity distribution is shown in Fig. 5.

On the other hand, the focus of positive diffraction orders is narrower and shorter. The location of diffraction order $n=1$ coincides with the analytical and numerical analysis. Also, orders $n=3,5$ are observed, but with low intensity.

Concluding, in this Letter we have analyzed the near-field behavior of chirped diffraction gratings with a linear dependence in the spatial frequency, $q(x)=q_{0}+q_{a} x$. The Fresnel approach has been used to obtain analytical expressions of the intensity distribution. When the chirped grating is illuminated with a Gaussian beam, we have found that the period and location of high-contrast fringes vary with the position $x$. We have also found that positive diffraction orders present focusing properties, with different width, position, and depth of focus of the beam waists. Numerical simulations based on the RayleighSommerfeld approximation have been carried out, as well as experimental verification. The analytical equations obtained in this work are in good agreement with the numerical simulations and experimental results. This formalism can be of interest in applications such as photonics and metrology.

204

205

206

207

208

209

210 211 212

213

214

215

216

217

218

219

220

221

222

223

224

225

226

227

228

229

230

231

Funding. Universidad Complutense de Madrid (UCM) 2232 (art. 83 LOU num. 52-2016).

\section{REFERENCES}

1. W. H. F. Talbot, Philos. Mag. 9(56), 401 (1836).

2. Lord Rayleigh, Philos. Mag. 11(67), 196 (1881).

3. J. Wen, Y. Zhang, and M. Xiao, Adv. Opt. Photon. 5, 83 (2013).

4. S. Szapiel and K. Patorski, J. Mod. Opt. 26, 439 (1979).

5. L. M. Sanchez-Brea, F. J. Torcal-Milla, and E. Bernabeu, Opt. Commun. 278, 23 (2007).

6. F. J. Torcal-Milla, L. M. Sanchez-Brea, and E. Bernabeu, Appl. Opt. 46, 3668 (2007).

7. F. J. Torcal-Milla, L. M. Sanchez-Brea, and E. Bernabeu, J. Opt. Soc. Am. A 25, 2390 (2008).

8. F. J. Torcal-Milla, L. M. Sanchez-Brea, and E. Bernabeu, Opt. Express 16, 19757 (2008).

9. J. M. Rico-Garca and L. M. Sanchez-Brea, Appl. Opt. 48, 3062 (2009).

10. W. D. Montgomery, J. Opt. Soc. Am. A 57, 772 (1967).

11. J. Azaña and M. A. Muriel, IEEE J. Sel. Top. Quantum Electron. 7, 728 (2001).

12. M. C. Hettrick, S. Bowyer, R. F. Malina, C. Martin, and S. Mrowka, Appl. Opt. 24, 1737 (1985).

13. W. R. McKinney, Rev. Sci. Instrum. 63, 1410 (1992).

14. N. Gao, H. Li, X. Zhu, Y. Hua, and C. Xie, Opt. Lett. 38, 2829 (2013).

15. F. Shen and A. Wang, Appl. Opt. 45, 1102 (2006). 


\section{Queries}

1. AU: In the sentence beginning "The camera is placed on a motorized ...," please spell out "PI."

2. AU: The funding information for this article has been generated using the information you provided to OSA at the time of article submission. Please check it carefully. If any information needs to be corrected or added, please provide the full name of the funding organization/institution as provided in the CrossRef Open Funder Registry (http://www.crossref.org/fundingdata/registry.html).

3. AU: Please verify my change to journal title in Ref. 4 . 